\title{
Facile fabrication of high-resolution extreme ultraviolet interference lithography grating masks using footing strategy during electron beam writing
}

\author{
Li Wang, ${ }^{a}$ ) Daniel Fan, Vitaliy A. Guzenko, and Yasin Ekinci \\ Laboratory for Micro- and Nanotechnology, Paul Scherrer Institute, CH-5232 Villigen PSI, Switzerland
}

(Received 7 July 2013; accepted 9 September 2013; published 25 September 2013)

\begin{abstract}
The authors demonstrate a method for facile fabrication of transmission grating masks used in extreme ultraviolet interference lithography (EUV-IL) based on a footing strategy during electron beam writing of mask gratings. By modifying the electron beam lithography pattern and dose distribution, a thin footing is generated between the mask grating lines. This thin footing layer effectively prohibits subsequent metal deposition using a standard electroplating setup in the grating region, while outside the grating region metal is electrodeposited freely, absorbing EUV radiation and serving as an EUV-IL mask grating central photon stop layer. The strategy the authors present greatly simplifies the mask fabrication process and increases the yield, as the crucial central stop region can be fabricated directly and without further overlay exposure steps either using electron beam or photolithography. () 2013 American Vacuum Society. [http://dx.doi.org/10.1116/1.4822016]
\end{abstract}

\section{INTRODUCTION}

The state-of-the-art in semiconductor manufacturing, based on optical double-patterning and immersion lithography using light sources with $193 \mathrm{~nm}$ wavelength, is approaching its resolution limit. ${ }^{1}$ Various methods in nanofabrication for high-resolution patterning are being pursued, including top-down methods such as nanoimprint lithography, electron beam lithography (EBL), and helium beam patterning, as well as bottom-up approaches such as directed self-assembly and epitaxial growth. ${ }^{2-4}$ Optical lithography using extreme ultraviolet (EUV) radiation at $13.5 \mathrm{~nm}$ wavelength has attracted interest from both industry and academia, as this method allows high throughput parallel processing of high-resolution features below $20 \mathrm{~nm}$, and is expected to be used for sub- $16 \mathrm{~nm}$ nodes. ${ }^{1,5,6}$ In parallel with projection optical systems developed for industrial applications, EUV interference lithography (EUV-IL) is used as a powerful tool for both scientific as well as industrial research. ${ }^{7,8} \mathrm{IL}$ at the EUV wavelength has not only been used to pattern sub-10 nm structures but is also used to develop photoresist technology and etching technology for semiconductor industries. ${ }^{9-11}$ As such, it is a powerful tool for scientific and industrial research, and dedicated EUV-IL tools have been set up using synchrotron, ${ }^{12}$ laser, ${ }^{13}$ and plasma sources. ${ }^{14}$

A typical EUV-IL scheme involves the use of transmission grating masks and the interference of their first order diffracted beams, as shown in Fig. 1. The aerial image pattern has a periodicity that is one half of the transmission grating periodicity. The incident beam on the area in between the two transmission gratings must be prevented from propagating toward the image plane, which will otherwise add strong background to the interference aerial image. Therefore, a photon central stop is required, complicating

${ }^{a)}$ Electronic mail: li.wang@psi.ch the mask fabrication process. For example, the mask gratings are typically fabricated using EBL on a 100-nm-thick silicon nitride membrane, where three complete lithographic processes are needed: first to make the second overlay markers, second to write the transmission gratings, and third to write the second overlay photon central stop area. Due to the fragile properties of the thin membranes, the processing of such EUV-IL masks has low yield. To this end, reducing the number of processing steps would enhance the success rate of mask fabrication and increase the yield. In this context, we demonstrate a simplified and nontraditional method for EUV-IL mask fabrication, which involves only one EBL writing process and can provide high-resolution EUV-IL masks.

\section{EXPERIMENTAL DESIGN, RESULTS, AND DISCUSSION}

A typical process flow for making EUV-IL masks is schematically shown in Fig. 2(a). Briefly, the mask was fabricated on a 100 -nm-thick $\mathrm{Si}_{3} \mathrm{~N}_{4}$ membrane, where the transmission gratings were written on by EBL. The photon central stop layer was generated by a combination of second overlay exposure with EBL and metal absorption layer deposition and lift-off. ${ }^{11}$ In our recent development, an improved method and process for the EUV-IL mask fabrication was introduced, which is schematically shown in Fig. 2(b). ${ }^{15}$ Such kinds of masks were fabricated on a 100-nm-thick $\mathrm{Si}_{3} \mathrm{~N}_{4}$ membrane with a thermally evaporated $\mathrm{Cr} / \mathrm{Au} / \mathrm{Cr}$ multilayer having a typically thickness of $2 / 5 / 2 \mathrm{~nm}$, respectively. The $\mathrm{Si}_{3} \mathrm{~N}_{4}$ membrane was spin-coated with negative tone hydrogen silsesquioxane (HSQ) resist (FOX 14, Dow Corning), which has a thickness of $200 \mathrm{~nm}$ at $3000 \mathrm{rpm}$ spincoating. The gratings were written by EBL and subsequently developed in a $1 \%$ aqueous solution of $\mathrm{NaOH}$. A chlorine dry etching process was then used to etch through the top $\mathrm{Cr}$ layer in order to facilitate the subsequent Au electroplating 


$$
p=\frac{\lambda}{2 \sin (\theta)}=\frac{g}{2 m} \quad \begin{aligned}
& \text { g: grating period } \\
& \text { m:diffraction order }
\end{aligned}
$$

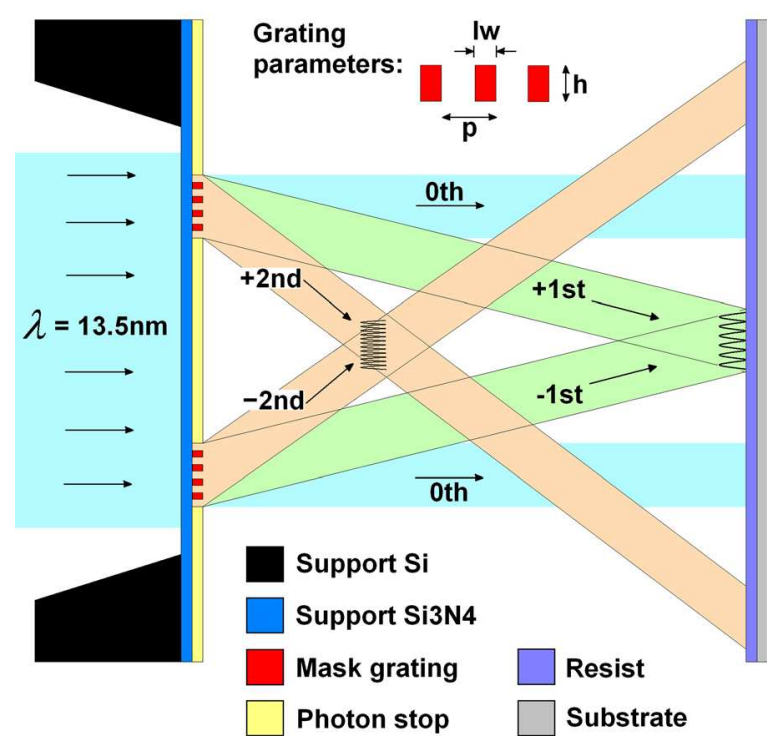

FIg. 1. (Color online) Schematic view of EUV-IL. Coherent light is transmitted through the diffraction gratings. The first-order diffraction beams interfere to produce an aerial pattern on the substrate, while the incident beam on to the area between the gratings is stopped by an opaque central stop.

of the grating bars to a thickness of approximately $150 \mathrm{~nm}$. The electroplating process of $\mathrm{Au}$ was optimized in order to obtain low-stress Au films, because of the limited mechanical stability of the membranes. Finally, the fabrication was completed by removal of HSQ in buffered oxide etcher. This mask fabrication process provides advantages over the aforementioned typical process, because it does not need a second overlay exposure with EBL and therefore does not need EBL markers prepatterned on membrane. However, when a high resolution EUV-IL mask was required, i.e., for grating periods in the sub- $100 \mathrm{~nm}$ range, the aspect ratio of the structures of either resist gratings or metal ones increased rapidly leading to the pattern collapse during the $\mathrm{N}_{2}$ blow drying of the samples. It was not possible to decrease the thickness of the photon central stop since it would increase the transmission of photons contributing to the background of the aerial image pattern. To overcome this problem, a supercritical drying method was implemented in the process flow, and the pattern collapse problem was successfully solved. However, we noticed another drawback in the subsequent metal plating process. We found that for grating lines below a certain period the electrodeposited gold was inhomogeneous or even absent between the grating lines [see scanning electron microscope (SEM) in Fig. 3].

In order to circumvent both pattern collapse and inhomogeneous metal deposition when fabricating high resolution EUV-IL masks using the process flow in Fig. 2(b) while still keeping a minimal number of steps compared to the process flow in Fig. 2(a), the strategy was developed to use the resist gratings written by EBL as the transmission gratings, followed by electroplating of metal central stop layer outside the grating area while simultaneously avoiding metal plating in between the lines, as schematically shown in Fig. 4. Making a close survey of the EUV optical properties of (a)

Deposition and
spin-coating
E-beam
lithography
Reactive ion
etching
PMMA
spin-coating
E-beam
lithography

Au deposition or electro-plating

Lift-off
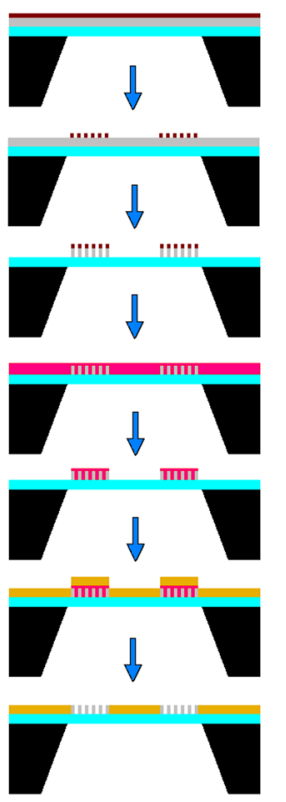

(b)
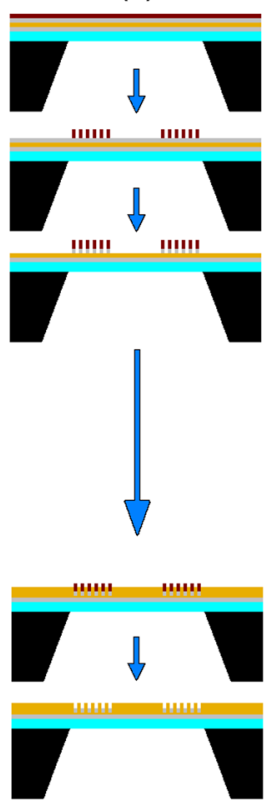

Au (c)
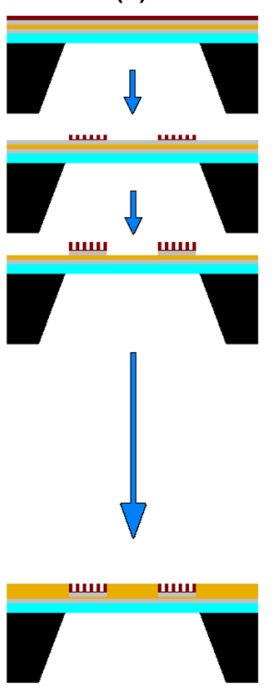

HSQ

PMMA

FIG. 2. (Color online) Schematic of process flows for producing diffraction gratings with on $\mathrm{Si}_{3} \mathrm{~N}_{4}$ membranes with central stop using different strategies. (a) Typical process flow for making EUV-IL mask with gold central stop layer using two times EBL. (b) Process by electroplating gold in between grating lines to produce gold gratings with gold central stop. (c) Process by using footings to prevent gold electroplating between grating lines, while gold is electroplated for the central stop. 


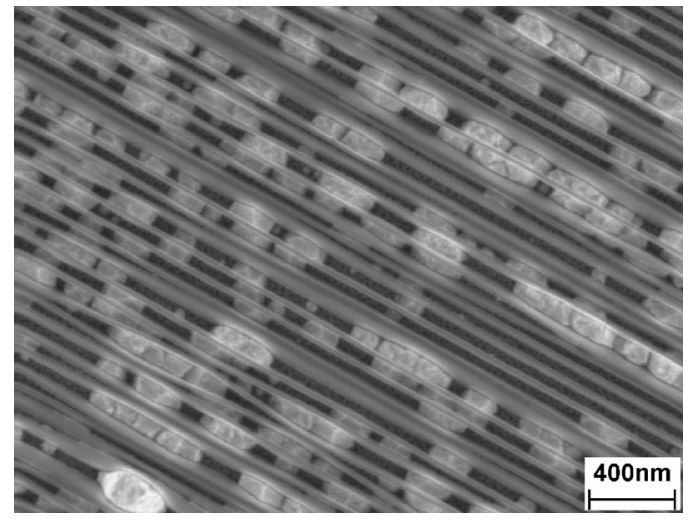

FIG. 3. SEM picture of failed gold electroplating between $50 \mathrm{~nm}$ wide lines of HSQ. The gold formed isolated grains and did not fill in the spaces homogeneously. The resulting structure is thus unsuitable to be used as a mask for EUV-IL.

various resists, some of them, such as HSQ and hafnium oxide based resists (from Inpria, Inc.), present sufficiently strong absorbance of EUV light, specifically at the $13.5 \mathrm{~nm}$ wavelength. Eventually, the possible modified process flow with minimum of fabrication steps of high-resolution EUVIL mask is schematically shown in Fig. 2(c), where in contrast to that in Fig. 2(b), there is no need in removing the resist template.

The resist patterning by EBL is the same as aforementioned in the experimental, and its thickness determines the grating diffraction order efficiency and the mask efficiency. As an example, Fig. 5 shows a simulated HSQ grating, with a pitch of $56 \mathrm{~nm}$, with calculated first order diffraction efficiency as a function of grating duty cycle and grating thickness (HSQ thickness). An efficiency of $6 \%$ for first order diffraction could be obtained with HSQ thickness of $80 \mathrm{~nm}$, and duty cycle of 0.5 . The challenge of this strategy comes from being able to electroplate the metal central stop layer outside the grating area, and simultaneously avoiding metal plating in between the lines. The most straightforward solution is to provide a buffer layer in between the grating area and the underneath metal electroplating conductive layer. To achieve this, an ultrathin layer of resist itself could be exposed before the transmission grating exposure. However, this pre-exposure would change the resist structure and lead to loss of resist resolution and contrast. ${ }^{16}$ Another way to generate a thin layer suppressing electroplating is to promote the footing of lines formed during the exposure. Practically,

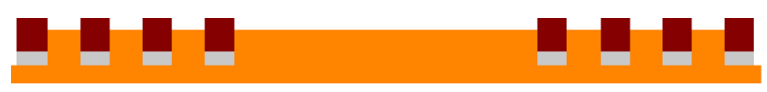

(a) without footing

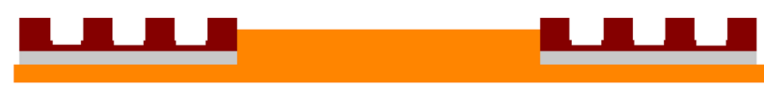

(b) with footing

FIG. 4. (Color online) Schematics illustrating the difference between the two processing schemes as shown in Figs. 2(b) and 2(c) in detail. (a): Without footing, gold is electroplated between the grating lines. (b): With footing, electroplating is inhibited and the spaces remain empty.

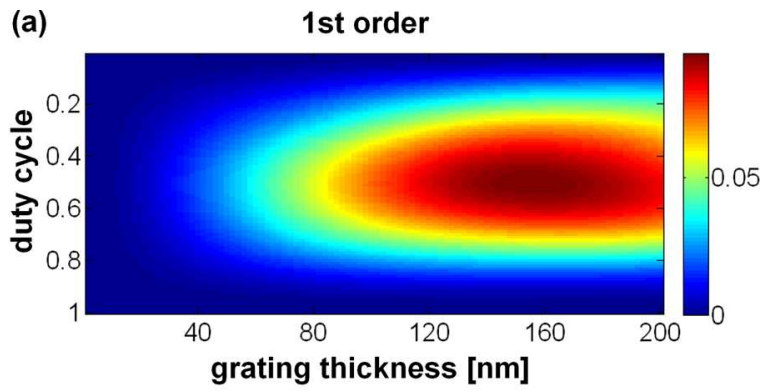

(b)

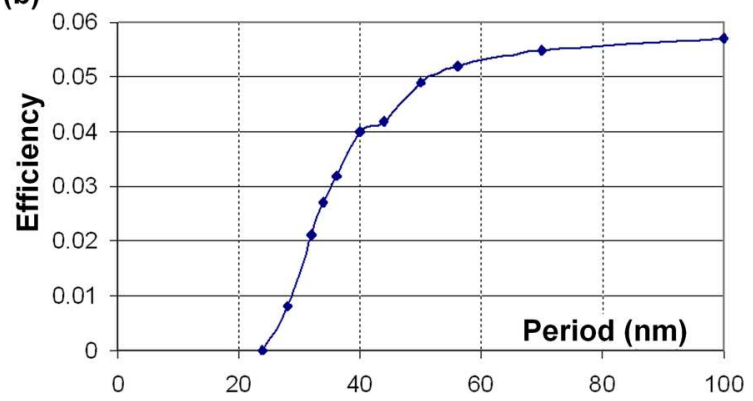

FIG. 5. (Color online) By using rigorous coupled wave analysis (RCWA) numerical simulation, (a) the first order diffraction efficiency map of HSQ grating with period $28 \mathrm{~nm}$ as a function of grating thickness and duty cycle; (b) the 1st order diffraction efficiency of HSQ grating with thickness of $80 \mathrm{~nm}$, duty cycle of 0.5 , as a function of grating period.

this was achieved by adding lines in between the grating lines, and exposing them at much lower dose. Here, the profile of this bottom thin layer for electroplating prohibition might be under question. To answer it, we performed an EBL simulation with the embedded module in e-beam exposure preparation software LayoutBEAMER (GenISys
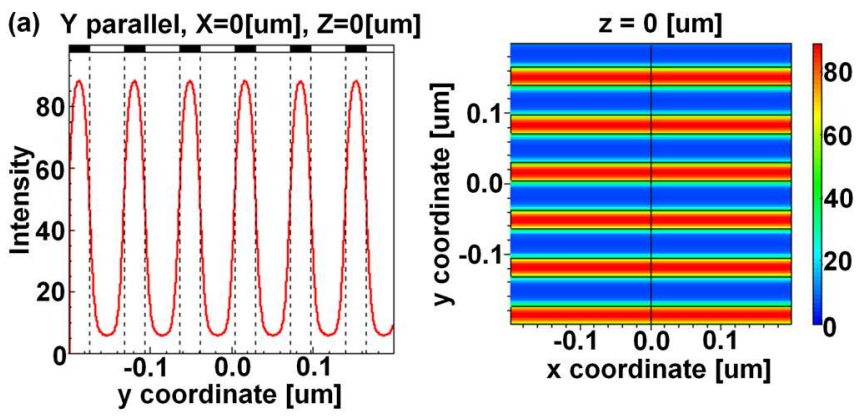

(b) $Y$ parallel, $X=0$ [um], $Z=0$ [um]

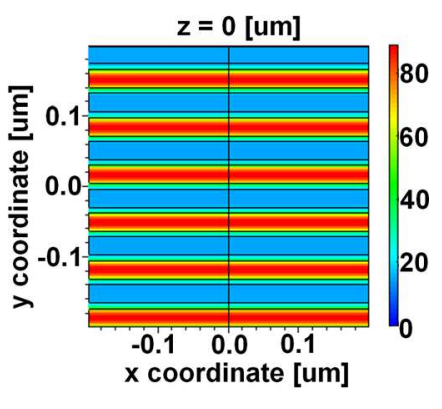

FIG. 6. (Color online) Cross-section profiles of the simulated e-beam dose for (a) standard grating and (b) grating with footing (calculations were done using modules of exposure preparation software LayoutBEAMER (GenISys $\mathrm{GmbH}$ ). By putting $10 \%$ of additional dose into the spaces between the lines, the shape of the grating was not considerably influenced, while proper conditions for the footing formation were created. 

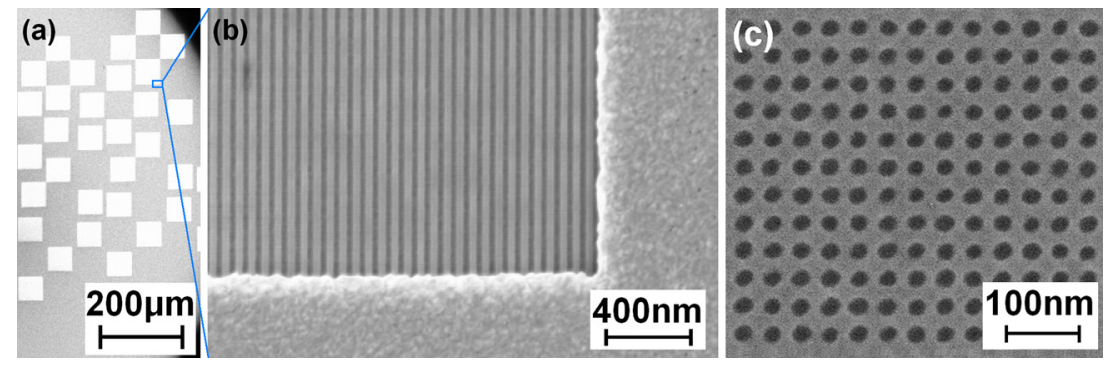

FIG. 7. (Color online) (a) SEM images of gold-electroplated membranes with gratings with footing. (b) Magnified image of box area in (a) Gold is absent in the grating regions while it is homogeneously electrodeposited elsewhere. (c) SEM images of developed JSR resist after EUV-IL exposure using a mask fabricated using EBL footing strategy.

$\mathrm{GmbH})$. The profiles of two different grating layouts were simulated: Type one had a standard lines/spaces design, whereas type two had extra lines in between the normal gratings of type one with the dose assigned to be small fraction of the base-dose. Figure 6 shows the simulated result of the dose distribution in the grating area. From the cross-sectional profile perpendicular to the lines, we observe a vertical shift of the dose intensities. There are no visible distortions or anomalies, such as spikes in between grating lines. The simulation shows that it is possible to produce grating footing while preserving the overall grating geometry by adding an extra line grating in between the transmission grating lines and optimizing the e-beam dose assignment for them.

To experimentally prove the simulations, a high resolution EUV-IL grating mask having groups of four gratings with pitches from 34 down to $26 \mathrm{~nm}$, arranged as shown in Fig. 7(a), was fabricated using an EBL tool Vistec EBPG5000Plus operated at $100 \mathrm{keV}$ electron energy. Following the exposure, samples were developed in a $1 \%$ aqueous solution of $\mathrm{NaOH}$ for $4 \mathrm{~min}$ and rinsed in DI water. A chlorine dry etching process was then used to etch through the top $\mathrm{Cr}$ layer in order to facilitate the subsequent Au electroplating of the photon central stop to a thickness of approximately $200 \mathrm{~nm}$ to effectively eliminate background of the aerial image pattern on wafer. The SEM image of a fabricated mask in Fig. 7(b) clearly indicates that the Au photon central stop layer, which is thicker than the HSQ gratings, was electroplated solely outside the grating area. Smooth and homogeneous HSQ gratings without any bridging in between were observed, in accordance with the EBL simulations. The EUV exposures were performed at the XIL-II beamline at the Swiss Light Source (SLS) with a spatially coherent EUV light of $13.5 \mathrm{~nm}$ wavelength and a bandwidth of $\Delta \lambda / \lambda=4 \%$. The patterns were recorded on Si wafers with an underlayer coated with a chemically amplified EUV resist (Japanese Synthetic Rubber Corporation) at a spin-coating speed of $3500 \mathrm{rpm}$ for $45 \mathrm{~s}$, resulting in a resist thickness of $22 \mathrm{~nm}$. The resist thin film was prebaked at $130^{\circ} \mathrm{C}$ for $60 \mathrm{~s}$. The exposure dose on mask level was varied between 20 and $200 \mathrm{~mJ} / \mathrm{cm}^{2}$. After EUV exposure, the wafer was baked at $110^{\circ} \mathrm{C}$ for $60 \mathrm{~s}$. Following the exposure, the samples were developed in a standard developer MF26A aqueous solution of tetramethylammonium hydroxide for $30 \mathrm{~s}$. The results are summarized in Fig. 7(c). Using a grating mask fabricated using the footing strategy, we obtained homogeneous contact holes with half pitch down to $20 \mathrm{~nm}$. This is a clear demonstration of successful utilization of developed technique for fabrication of mask for EUV-IL with high quality gratings and negligible central stop leakage.

\section{CONCLUSIONS}

In conclusion, we have demonstrated a simple technique for fabricating high-resolution transmission grating masks to be used in EUV-IL. This involves adding an extra line in between the grating lines and modifying the e-beam dose profile so that grating footings are realized. We designed EBL exposure parameters by simulation with embedded modules in EBL exposure preparation software, considering the feasible nanofabrication processes. These successfully obtained footings are acting as a thin film prohibiting etching of the top Cr layer and consequent electroplating of gold in the grating region while the central stop region is electroplated. We demonstrated the feasibility of this technique using HSQ, which, due to its high resolution and sufficient EUV absorbance, is a suitable candidate for fabricating EUV-IL grating masks. Using such masks, we presented exposed by EUV-IL and developed feature sizes down to $20 \mathrm{~nm}$ in, a chemical amplified resist, using EUV-IL and mask written by HSQ gratings with the footing strategy. Further optimization of the mask fabrication and resist processing will be explored in order to achieve sub- $10 \mathrm{~nm}$ contact hole features.

\section{ACKNOWLEDGMENTS}

The authors thank Michaela Vockenhuber for technical support. This project was funded by the Nano-Argovia programme of the Swiss Nanoscience Institute Basel (SNI). Part of this work was performed at the SLS, Paul Scherrer Institute, 5232 Villigen PSI, Switzerland.

${ }^{1}$ International Technology Roadmap for Semiconductors, see: http:// www.itrs.net/.

${ }^{2}$ V. Sidorkin, E. van Veldhoven, E. van der Drift, P. Alkemade, H. Salemink, and D. Maas, J. Vac. Sci. Technol. B 27, L18 (2009).

${ }^{3}$ Y. S. Jung, J. B. Chang, E. Verploegen, K. K. Berggren, and C. A. Ross, Nano Lett. 10, 1000 (2010).

${ }^{4}$ A. Tavakkoli, K. G., S. N. Piramanayagam, M. Ranjbar, R. Sbiaa, and T.

C. Chong, J. Vac. Sci. Technol. B 29, 011035 (2011).

${ }^{5}$ C. Wagner and N. Harned, Nature Photon. 4, 24 (2010).

${ }^{6}$ G. Tallents, E. Wagenaars, and G. Per, Nature Photon. 4, 809 (2010).

${ }^{7}$ H. H. Solak, J. Phys. D: Appl. Phys. 39, R171 (2006). 
${ }^{8}$ V. Auzelyte et al., J. Micro/Nanolith. MEMS MOEMS 8, 021204 (2009).

${ }^{9}$ K. Petrillo et al., J. Vac. Sci. Technol. B 25, 2490 (2007).

${ }^{10}$ R. Gronheid, H. H. Solak, Y. Ekinci, A. Jouve, and F. Van Roey, Microelectron. Eng. 83, 1103 (2006).

${ }^{11}$ B. Päivanranta, A. Langner, E. Kirk, C. David, and Y. Ekinci, Nanotechnology 22, 375302 (2011).

${ }^{12}$ J. Wallace, Y. C. Cheng, A. Isoyan, Q. Leonard, M. Fisher, M. Green, J. Bisognano, P. F. Nealey, and F. Cerrina, Nucl. Instrum. Methods Phys. Res. A 582, 254 (2007).
${ }^{13}$ A. Ritucci, A. Reale, P. Zuppella, L. Reale, P. Tucceri, G. Tomassetti, P. Bettotti, and L. Pavesi, J. Appl. Phys. 102, 034313 (2007).

${ }^{14}$ M. Goldstein, S. H. Lee, Y. A. Shroff, P. J. Silverman, D. Williams, H. Park, M. A. Piestrup, and R. H. Pantell, in Proceedings of the 2005 FEL Conference (2005), p. 422.

${ }^{15}$ L. Wang, B. Terhalle, M. Hojeij, V. A. Guzenko, and Y. Ekinci, J. Vac. Sci. Technol. B 30, 031603 (2012).

${ }^{16}$ V. Sidorkin, E. van der Drift, and H. Salemink, J. Vac. Sci. Technol. B 26, 2049 (2008). 